\title{
Quantum Charged Particle in a Flat Box under Static Electromagnetic Field with Landau's Gauge and Special Case with Symmetric Gauge
}

\author{
Gustavo V. López, Jorge A. Lizarraga, Omar J. P. Bravo \\ Departamento de Física, Universidad de Guadalajara, Guadalajara, México \\ Email: gulopez@cencar.udg.mx,yjorge.a.lizarraga.b@gmail.com,zomarbravomac@icloud.com
}

How to cite this paper: López, G.V., Lizarraga, J.A. and Bravo, O.J.P. (2021) Quantum Charged Particle in a Flat Box under Static Electromagnetic Field with Landau's Gauge and Special Case with Symmetric Gauge. Journal of Modern Physics, 12, 1464-1474.

https://doi.org/10.4236/jmp.2021.1210088

Received: July 25, 2021

Accepted: August 21, 2021

Published: August 24, 2021

Copyright $\odot 2021$ by author(s) and Scientific Research Publishing Inc. This work is licensed under the Creative Commons Attribution International License (CC BY 4.0).

http://creativecommons.org/licenses/by/4.0/

\begin{abstract}
We summarize our results about the quantization of a charged particle motion without spin inside a flat box under a static electromagnetic field with Landau's gauge for the magnetic field, where Fourier's transformation was used to analyze the problem, to point out that there exists a wave function which is different to that one given by Landau with the same Landau's levels. The quantization of the magnetic flux is deduced differently to previous one, and a new solution is presented for the case of symmetric gauge of the magnetic field, and having the same Landau's levels.
\end{abstract}

\section{Keywords}

Landau's Gauge, Symmetric Gauge, Quantum Hall Effect, Flat Box

\section{Introduction}

The work of Klitzing, Dora and Pepper [1] presented a breakthrough in experimental physics due to its success in measuring the Hall voltage of a twodimensional electron gas realized in a MOSFET. The important fact discovered in this experiment was that the Hall resistance is quantized, and Landau's eigenvalues solution [2] (Landau's levels) of a charged particle in a flat surface with magnetic field has become of great importance in trying to understand integer Hall effect [1] [3] [4] [5] [6], fractional Hall effect [6] [7] [8] [9], and topological insulators [10]-[14]. This last elements promise to become essential for future nanotechnology devices [15] [16] [17]. Therefore, it is worth to re-take this problem and to consider in detail the characteristics that it presents. In our previous paper [18], we considered the static magnetic field given through the Lan- 
dau's gauge and obtained, by using the Fourier transformation, a different solution for the eigenfunction to those given by Landau. We summarize those results here and make a different approach to obtain the quantization of the magnetic flux or the density of states between two Landau's levels. We continue considering that this result could be relevant, because Landau's solution is kept using in different works like Prange's [19], Laughling's [20], solid state and quantum transport books as well [3] [7] [21] [22]. In addition, for the especial case where the charged particle is moving on the plane $\mathrm{x}-\mathrm{y}$ under the same static transversal magnetic field but defined by the symmetric gauge, we present a new solution, which matches the characteristics mentioned in [18] and on this paper, we have the same Landau's Levels as solution of the eigenvalue problem.

\section{Analytical Approach with Landau's Gauge}

Let us consider a charged particle " $q$ " with mass " $m$ " in the box with a constant magnetic field orthogonal to the flat surface $\mathrm{x}-\mathrm{y}, \boldsymbol{B}=(0,0, B)$, where the magnetic field is given in terms of the vector potential $\boldsymbol{A}, \boldsymbol{B}=\nabla \times \boldsymbol{A}$, and let us choose the Landau's gauge $\boldsymbol{A}=(-B y, 0,0)$ to represents this magnetic field.

\subsection{Analytical Approach for the Case $B=(0,0, B)$}

For a nonrelativistic charged particle, the Hamiltonian of the system (units CGS) is

$$
H=\frac{(\boldsymbol{p}-q \boldsymbol{A} / \boldsymbol{c})^{2}}{2 m},
$$

where $\boldsymbol{p}$ is the generalized linear momentum, $\boldsymbol{A}$ is the magnetic potential, and " $c$ " is the speed of light. Therefore, the Hamiltonian has the following form $H=\frac{\left(p_{x}+q B y / c\right)^{2}}{2 m}+\frac{p_{y}^{2}}{2 m}+\frac{p_{z}^{2}}{2 m}$, which does not depend explicitly on time and the eigenvalue problem, $\hat{H} \Phi=E \Phi$, for the Schrödinger's equation [23] is

$$
\left\{\frac{1}{2 m}\left(\hat{p}_{x}^{2}+\frac{2 q B}{c} y \hat{p}_{x}+\frac{q^{2} B^{2}}{c^{2}} y^{2}\right)+\frac{\hat{p}_{y}^{2}}{2 m}+\frac{\hat{p}_{z}^{2}}{2 m}\right\} \Phi=E \Phi .
$$

The variable " $Z$ " is separable through the proposition $\Phi(\boldsymbol{x})=\phi(x, y) \mathrm{e}^{-i k_{z} z}, k_{z} \in \Re$, resulting in the following equation

$$
\left\{\frac{1}{2 m}\left(\hat{p}_{x}^{2}+\frac{2 q B}{c} y \hat{p}_{x}+\frac{q^{2} B^{2}}{c^{2}} y^{2}\right)+\frac{\hat{p}_{y}^{2}}{2 m}\right\} \phi=E^{\prime} \phi,
$$

where $E^{\prime}$ is

$$
E^{\prime}=E-\frac{\hbar^{2} k_{z}^{2}}{2 m} .
$$

Solving this equation through Fourier transformation [24] on the variable " $x$ ", $\hat{\phi}(k, y)=\mathcal{F}[\phi]=\frac{1}{\sqrt{2 \pi}} \int_{\Re} \mathrm{e}^{i k x} \phi(x, y) \mathrm{d} x$, it is known [18] (Equation (18)) that one 
gets the solution

$$
\left.\Phi_{n, k_{z}}(\boldsymbol{x})=\frac{1}{\sqrt{L_{y} L_{z}}}\left(\frac{m \omega_{c}}{\hbar}\right)^{1 / 4} \mathrm{e}^{-i\left(\frac{m \omega_{c}}{\hbar} x y+k_{z} z\right.}\right) \psi_{n}\left(\sqrt{\frac{m \omega_{c}}{\hbar}} x\right) .
$$

and

$$
E_{n, k_{z}}=\hbar \omega_{c}\left(n+\frac{1}{2}\right)+\frac{\hbar^{2} k_{z}^{2}}{2 m} .
$$

where $\psi_{n}$ represents the solution of the quantum harmonic oscillator, and $\omega_{c}$ is the so called cyclotron frequency

$$
\omega_{c}=\frac{q B}{m c}
$$

These eigenvalues represent just the Landau's levels, but its solution (5) is different to that given by Landau on the variables " $x$ " and " $y$ ". Note that there is not displacement at all in the harmonic oscillation solution. Now, assuming a periodicity in the $z$-direction, $\Phi_{n, k_{z}}(\boldsymbol{x}, t)=\Phi_{n, k_{z}}\left(x, y, z+L_{z}, t\right)$, the usual condition $k_{z} L_{z}=2 \pi n^{\prime}, n^{\prime} \in \mathcal{Z}$ makes the eigenvalues to be written as and the general solution of Schrödinger's equation can be written as ${ }^{1}$

$$
\Phi_{n, n^{\prime}}(\boldsymbol{x})=\frac{1}{\sqrt{L_{y} L_{z}}}\left(\frac{m \omega_{c}}{\hbar}\right)^{1 / 4} \mathrm{e}^{-i\left(\frac{m \omega_{c}}{\hbar} x y+\frac{2 \pi n^{\prime}}{L_{z}} z\right)} \psi_{n}\left(\sqrt{\frac{m \omega_{c}}{\hbar}} x\right) .
$$

and

$$
E_{n, n^{\prime}}=\hbar \omega_{c}(n+1 / 2)+\frac{\hbar^{2} 2 \pi^{2}}{m L_{z}^{2}} n^{\prime 2},
$$

On the other hand, we could have used the boundary conditions $\Phi_{n, k_{z}}(x, y, 0)=\Phi_{n, k_{z}}\left(x, y, L_{z}\right)=0$ to obtain the same expression (9) but with the following eigenfunction

$$
\Phi_{n, n^{\prime}}(\boldsymbol{x})=\frac{1}{\sqrt{L_{y} L_{z}}}\left(\frac{m \omega_{c}}{\hbar}\right)^{1 / 4} \mathrm{e}^{-i\left(\frac{m \omega_{c}}{\hbar} x y\right)} \sin \left(\frac{2 \pi n^{\prime}}{L_{z}} z\right) \psi_{n}\left(\sqrt{\frac{m \omega_{c}}{\hbar}} x\right)
$$

It is necessary to point out that the solution (10) is not separable solution type on the variables $x$ and $y$, contrary to Landau's solution. In addition, harmonic oscillator is on the variable $x$ without displacement, contrary to Landau's solution which the harmonic oscillator is on the variable y with a displacement. Now, the area of the surface of a circular ring of radius $r_{1}$ and $r_{2}$ is given classically by $\Delta A=\pi\left(r_{2}^{2}-r_{1}^{2}\right)$, where $r^{2}=x^{2}+y^{2}$ and $r_{2}>r_{1}$. For the quantum case and using (8), one has

${ }^{1}$ In fact, our Hamiltonian is invariant under translation in the $\mathrm{x}$-direction, and this fact is represented by $\left[\hat{p}_{x}, \hat{H}\right]=0$, where $\hat{p}_{x}$ is the infinitesimal generator of the element of the group of symmetry, it is not difficult to see that $\hat{p}_{x} \Phi_{n, k_{z}}=m \omega_{c}(i x-y) \Phi_{n, k_{z}}-2 n i \hbar \sqrt{\frac{m \omega_{c}}{\hbar}} \Phi_{n-1, k_{z}}$ is another eigenfunction of our Hamiltonian, $\hat{H}\left(\hat{p}_{x} \Phi_{n, k_{z}}\right)=E_{n, n^{\prime}}\left(\hat{p}_{x} \Phi_{n, k_{z}}\right)$. In this way, one has that the energy being double degenerated by this symmetry. The same will happen with the next cases. 


$$
\Delta A=\pi\left[\left\langle\Phi_{n_{2}, n_{2}^{\prime}}\left|r^{2}\right| \Phi_{n_{2}, n_{2}^{\prime}}\right\rangle-\left\langle\Phi_{n_{1}, n_{1}^{\prime}}\left|r^{2}\right| \Phi_{n_{1}, n_{1}^{\prime}}\right\rangle\right]=\frac{2 \pi \hbar}{m \omega_{c}}\left(n_{2}-n_{1}\right),
$$

where the integration has been carried out on the region $z \in\left[0, L_{z}\right]$, $y \in\left[-L_{y}, L_{y}\right]$ and $x \in(-\infty,+\infty)$. Now, since $\omega_{c}=q B / m c$, the above expression brings about the relation

$$
\frac{q B(\Delta A)}{2 \pi \hbar c}=n_{2}-n_{1}=j \in \mathcal{Z}
$$

which represents the quatization of the magnetic flux [18] (expression (20)), and it is related with the density of states between two Landau's levels [2]. If $\Phi=B(\Delta A)$ is the magnetic flux, and $\Phi_{0}=2 \pi \hbar c / 2 q$ is the so called quantum magnetic flux [25] [26], this expression can be written as

$$
\frac{\Phi}{\Phi_{0}}=2 j, \quad j \in \mathcal{Z}
$$

Thus, the general solution (absorbing the sign in the constants) is

$$
\Psi(\boldsymbol{x}, t)=\frac{1}{\sqrt{L_{y} L_{z}}}\left(\frac{m \omega_{c}}{\hbar}\right)^{1 / 4} \mathrm{e}^{-i \frac{m \omega_{c}}{\hbar} x y} \sum_{n, n^{\prime}} C_{n n^{\prime}} \mathrm{e}^{i \frac{2 \pi n^{\prime}}{L_{z}} z} \mathrm{e}^{-i \frac{E_{n, n^{\prime}}}{\hbar} t} \psi_{n}\left(\sqrt{\frac{m \omega_{c}}{\hbar}} x\right) .
$$

where the constants $C_{n n^{\prime}}$ must satisfy that $\sum_{n, n^{\prime}}\left|C_{n n^{\prime}}\right|^{2}=1$. The Landau's levels $E_{n, n^{\prime}}$ are given by expression (9).

\subsection{The Analytical Approach for Case $B \perp E$}

The magnetic is given as before and electric constant fields is given by $\boldsymbol{E}=(0, \mathcal{E}, 0)$, and $\phi=-\mathcal{E} y$. Then, our Hamiltonian is [20] [21] [22]

$$
\hat{H}=\frac{\left(\hat{\boldsymbol{p}}-\frac{q}{c} \boldsymbol{A}\right)^{2}}{2 m}+q \phi(\boldsymbol{x}),
$$

and using again the Fourier transformation on the Schrödinger's equation, $i \hbar \frac{\partial \Psi}{\partial t}=\hat{H} \Psi$, it is known [18] (Equation (39)) that a solution is given by

$$
\Psi_{n, k_{z}}(\boldsymbol{x}, t)=\frac{1}{\sqrt{L_{y} L_{z}}}\left(\frac{m \omega_{c}}{\hbar}\right)^{1 / 4} \mathrm{e}^{-i \phi_{n, k_{z}}(\boldsymbol{x}, t)} \psi_{n}\left(\sqrt{\frac{m \omega_{c}}{\hbar}}\left(x-\frac{c \mathcal{E} t}{B}\right)\right)
$$

where the phase $\phi_{n, k_{z}}(\boldsymbol{x}, t)$ has been defined as

$$
\begin{aligned}
\phi_{n, k_{z}}(\boldsymbol{x}, t)= & {\left[\hbar \omega_{c}(n+1 / 2)+\frac{\hbar^{2} k_{z}^{2}}{2 m}-\frac{m c^{2} \mathcal{E}^{2}}{2 B^{2}}\right] \frac{t}{\hbar}-k_{z} z } \\
& +\frac{q B}{\hbar c}\left(x-\frac{c \mathcal{E} t}{B}\right)\left(y-\frac{m c^{2} \mathcal{E}}{q B^{2}}\right) .
\end{aligned}
$$

asking for the periodicity with respect the variable " $z$ ", $\Psi_{n, k_{z}}(\boldsymbol{x}, t)=\Psi_{n, k_{z}}\left(z, y, z+L_{z}, t\right)$, it follows that $k_{z} L_{z}=2 \pi n^{\prime}$ where $n^{\prime}$ is an integer number, and the above phase is now written as 


$$
\begin{aligned}
\phi_{n n^{\prime}}(\boldsymbol{x}, t)= & {\left[\hbar \omega_{c}(n+1 / 2)+\frac{\hbar^{2} 2 \pi^{2} n^{\prime 2}}{m L_{z}^{2}}-\frac{m c^{2} \mathcal{E}^{2}}{2 B^{2}}\right] \frac{t}{\hbar}-\frac{2 \pi n^{\prime}}{L_{z}} z } \\
& +\frac{q B}{\hbar c}\left(x-\frac{c \mathcal{E} t}{B}\right)\left(y-\frac{m c^{2} \mathcal{E}}{q B^{2}}\right) .
\end{aligned}
$$

Note from this expression that the term $\mathrm{e}^{-i \phi(x, t)}$ contains the element $\mathrm{e}^{i \frac{q B}{\hbar c} x y}$ which characterizes the non separability of the solution with respect these coordinates. Using the same arguments as before (11) to calculate the magnetic flux crossing an area $\Delta A$, one gets

$$
\frac{q B(\Delta A)}{\hbar c}=2 \pi j, \quad j \in \mathcal{Z},
$$

obtaining the same expression as (12). In this way, from these relations and the expression (16) we have a family of solutions $\left\{\Psi_{n n^{\prime}}(x, t)\right\}_{n, n^{\prime} \in \mathcal{Z}}$ of the Schrödinger equation,

$$
\Psi_{n n^{\prime}}(\boldsymbol{x}, t)=\frac{1}{\sqrt{L_{y} L_{z}}}\left(\frac{m \omega_{c}}{\hbar}\right)^{1 / 4} \mathrm{e}^{-i \phi_{n n^{\prime}}(\boldsymbol{x}, t)} \psi_{n}\left(\sqrt{\frac{m \omega_{c}}{\hbar}}\left(x-\frac{2 \pi j \hbar c^{2}}{q(\Delta A)} \mathcal{E} t\right)\right) .
$$

Now, by the same arguments we did in the previous case, the general solution would be written of the form

$$
\Psi(\boldsymbol{x}, t)=\sum_{n, n^{\prime}} \tilde{C}_{n n^{\prime}} \Psi_{n n^{\prime}}(\boldsymbol{x}, t),
$$

where one must have $\sum_{n, n^{\prime}}\left|\tilde{C}_{n n^{\prime}}\right|^{2}=1$.

\subsection{The Analytical Approach for Case $B \| E$}

The fields are of form $\boldsymbol{B}=(0, B, 0)$ and $\boldsymbol{E}=(0, \mathcal{E}, 0)$. The scalar and vector potentials are chosen as $\boldsymbol{A}=(B z, 0,0)$ and $\phi=-\mathcal{E} y$. The Shrödinger equation is for this case as

$$
i \hbar \frac{\partial \Psi}{\partial t}=\left\{\frac{\left(\hat{p}_{x}-q B z / c\right)^{2}}{2 m}+\frac{\hat{p}_{y}^{2}}{2 m}+\frac{\hat{p}_{z}^{2}}{2 m}-q \mathcal{E} y\right\} \Psi,
$$

the eigenvalue problem is now defined by the equation

$$
E \Phi=-\frac{\hbar^{2}}{2 m} \frac{\partial^{2} \Phi}{\partial x^{2}}+i \frac{q B \hbar z}{m c} \frac{\partial \Phi}{\partial x}+\frac{q^{2} B^{2}}{2 m c^{2}} z^{2} \Phi-\frac{\hbar^{2}}{2 m} \frac{\partial^{2} \Phi}{\partial y^{2}}-\frac{\hbar^{2}}{2 m} \frac{\partial^{2} \Phi}{\partial z^{2}}-q \mathcal{E} y \Phi .
$$

Using again the Fourier transform on the $\mathrm{x}$-variable, it is known [18] (Equation (55)) that one gets the following solution

$$
\Phi_{n, n^{\prime}}(\boldsymbol{x})=a_{n^{\prime}} \frac{1}{\sqrt{L_{y} L_{z}}}\left(\frac{m \omega_{c}}{\hbar}\right)^{1 / 4} \mathrm{e}^{-i \frac{m \omega_{c}}{\hbar} z x} \psi_{n}\left(\sqrt{\frac{m \omega_{c}}{\hbar} x}\right) \operatorname{Ai}\left(l^{-1}\left(y-y_{n^{\prime}}\right)\right) .
$$

where $\phi_{n}$ is the solution of the quantum harmonic oscillator, $A i$ is the Airy function [27], and $a_{n^{\prime}}$ is its normalization constant $a_{n^{\prime}}=1 /\left|A i^{\prime}\left(-l^{-1} y_{n^{\prime}}\right)\right|$.

Now, using the same arguments as before (11), but with $n_{1}^{\prime}=n_{2}^{\prime}$ (due to Airy functions) to calculate the magnetic flux crossing an area $\Delta A$, one gets 


$$
\frac{q B(\Delta A)}{\hbar c}=2 \pi j, \quad j \in \mathcal{Z},
$$

obtaining the same expression as (12). Then, we have obtained a family of solution of the Schrödinger Equation (22),

$$
\Psi_{n, n^{\prime}}(\boldsymbol{x}, t)=\mathrm{e}^{-i E_{n, n^{t}} / \hbar} \Phi_{n n^{\prime}}(\boldsymbol{x}),
$$

where the energies $E_{n, n^{\prime}}$ are given by $E_{n, n^{\prime}}=\hbar \omega_{c}(n+1 / 2)-q \mathcal{E} y_{n^{\prime}}$ with $y_{n^{\prime}}=l \tilde{\xi}_{n^{\prime}}$ and $\operatorname{Ai}\left(-\xi_{n^{\prime}}\right)=0$. The general solution of (22) can be written as

$$
\Psi(\boldsymbol{x}, t)=\mathrm{e}^{-i \frac{m \omega_{c}}{\hbar} x z} \sum_{n, n^{\prime}} \tilde{C}_{n, n^{\prime}} \mathrm{e}^{-i E_{n, n^{\prime}} / \hbar} \tilde{u}_{n, n^{\prime}}(x, y),
$$

with the condition $\sum_{n, n^{\prime}}\left|\tilde{C}_{n, n^{\prime}}\right|^{2}=1$, and where it has been defined the functions $\tilde{u}_{n, n^{\prime}}$ as

$$
\tilde{u}_{n, n^{\prime}}(x, y)=\frac{a_{n^{\prime}}}{\sqrt{L_{y} L_{z}}}\left(\frac{m \omega_{c}}{\hbar}\right)^{1 / 4} \psi_{n}\left(\sqrt{\frac{m \omega_{c}}{\hbar}} x\right) A i\left(l^{-1}\left(y-y_{n^{\prime}}\right)\right) .
$$

\section{Analytical Approach with Symmetric Gauge}

It is known that the selection of the gauge is not unique, there is always a transform of the form $\boldsymbol{A}_{L}=\boldsymbol{A}_{S}+\nabla \chi$, where $\boldsymbol{A}_{L}=(B y, 0)$ is the Landau's gauge and $\boldsymbol{A}_{S}=\frac{B}{2}(y,-x)$ is the symmetric gauge, and the eigenvalue equation limited on the plane $x$ - $y$ is written as follows

$$
E \Psi=\frac{1}{2 m}\left(\hat{p}_{x}^{2}+\hat{p}_{y}^{2}\right) \Psi+\frac{q B}{2 m c} \hat{L}_{z} \Psi+\frac{q^{2} B^{2}}{8 m c^{2}}\left(x^{2}+y^{2}\right) \Psi,
$$

where $\hat{L}_{z}$ is the z-component of the angular momentum operator, $\hat{L}_{z}=x \hat{p}_{y}-y \hat{p}_{x}$. This equation cannot be separated in cartesians or polar coordinates. Let us now define a complex variables $z=x+i y$ and $z^{*}=x-i y$, and the constants

$$
\varepsilon=\frac{m E}{2 \hbar^{2}} \quad \text { and } \quad \alpha=\frac{q B}{4 \hbar c},
$$

Equation (29) now takes the form of

$$
\varepsilon \Psi=-\frac{\partial^{2} \Psi}{\partial_{z} \partial_{z^{*}}}+\alpha\left(z \frac{\partial}{\partial z}-z^{*} \frac{\partial}{\partial_{z^{*}}}\right) \Psi+\alpha^{2} z z^{*} \Psi .
$$

Proposing a solution of the form

$$
\Psi\left(z, z^{*}\right)=\mathrm{e}^{-\alpha z z^{*}} \Phi\left(z, z^{*}\right)
$$

in the Equation (31), the resulting equation for the function $\Phi$ is

$$
\frac{\partial^{2} \Phi}{\partial z \partial z^{*}}-2 \alpha z \frac{\partial \Phi}{\partial z}=(\alpha-\varepsilon) \Phi .
$$

this last expression can be separated. So, let us chose $\Phi\left(z, z^{*}\right)=f(z) g\left(z^{*}\right)$, and by substituting, dividing by $f^{\prime}(z) g\left(z^{*}\right)$, and making some arrangements, 
it follows that

$$
\frac{g^{\prime}\left(z^{*}\right)}{g\left(z^{*}\right)}=2 \alpha z+(\alpha-\varepsilon) \frac{f(z)}{f^{\prime}(z)},
$$

since the expression on the left hand side of the above equality has a different mapping in the complex plane than the one on the right hand side, the latter expression will be generally satisfied if both of them are equal to a complex constant $\lambda \in \mathbb{C}$, and we obtain the next couple of equation

$$
\begin{gathered}
(2 \alpha z+\lambda) f^{\prime}(z)+(\alpha-\varepsilon) f(z)=0 . \\
g^{\prime}\left(z^{*}\right)=-\lambda g\left(z^{*}\right),
\end{gathered}
$$

The solution of Equation (36) is straight forward and is given by

$$
g\left(z^{*}\right)=B e^{-\lambda z^{*}}
$$

where $B$ is an arbitrary complex constant. Now, we search for integer complex solution of the expression Equation (35), writing the function as a power series of the variable $Z$

$$
f(z)=\sum_{k=0}^{\infty} a_{k} z^{k}
$$

substituting in Equation (35) and making some rearrangements, it follows that

$$
\sum_{k=0}^{\infty}\left[a_{k}(2 \alpha k+\alpha-\varepsilon)+a_{k+1}(k+1) \lambda\right] z^{k}=0,
$$

which brings about the following recurrent relation

$$
a_{k+1}=-\frac{2 \alpha k+\alpha-\varepsilon}{\lambda(k+1)} a_{k}
$$

Let us notice that we have the following asymptotic behavior

$$
\frac{a_{k+1}}{a_{k}} \underset{k \gg 1}{\longrightarrow} \frac{2 \alpha}{\lambda} \text {. }
$$

This means that there exist a natural number $N$ such that for $k \geq N$ we have that $a_{k+1}=a_{N}\left(\frac{2 \alpha}{\lambda}\right)^{k}$. Therefore, one would have the series

$$
\sum_{k=0}^{N} a_{k} z^{k}+a_{N} \sum_{k=N+1}^{\infty}\left(\frac{2 \alpha z}{\lambda}\right)^{k}
$$

which diverges for any value such that $|2 \alpha z| /|\lambda|>1$. In this way, one must cut the series and obtain a polynomial. To obtain a polynomial solution, we must demand that at some integer number $k=n$, one must have that $a_{n+1}=0$, and this implies that $2 \alpha n+\alpha-\varepsilon=0$, or

$$
\frac{\varepsilon_{n}-\alpha}{2 \alpha}=n \in \mathbb{Z}
$$

Thus, one can obtain that the energies of the system as 


$$
E_{n}=\hbar \omega_{c}\left(n+\frac{1}{2}\right)
$$

Note that the direct integration of Equation (35) gives us the solution

$$
f(z)=A(2 \alpha z+\lambda)^{(\varepsilon-\alpha) / 2 \alpha},
$$

where $A$ and $\lambda$ are in general complex constants. Using the expression (43), this function is written as

$$
f(z)=A(2 \alpha z+\lambda)^{n}=A \sum_{k=0}^{n}\left(\begin{array}{l}
n \\
k
\end{array}\right) \lambda^{n-k}(2 \alpha)^{k} z^{k},
$$

where $\left(\begin{array}{l}n \\ k\end{array}\right)=\frac{n !}{k !(n-k) !}$ is the binomial number. Therefore, using the expressions (46), (37), and (32), the solution of Equation (31) is

$$
\Psi_{n}\left(z, z^{*}\right)=A_{n} \mathrm{e}^{-\alpha z z^{*}-\lambda z^{*}}(2 \alpha z+\lambda)^{n},
$$

where $A_{n}$ is the normalized constant given by

$$
A_{n}=\mathrm{e}^{-|\lambda|^{2} / 4 \alpha} /(2 \alpha)^{\frac{n-1}{2}} \sqrt{\sum_{k=0}^{n}\left(\begin{array}{l}
n \\
k
\end{array}\right) \Gamma\left(n-k+\frac{1}{2}\right) \Gamma\left(k+\frac{1}{2}\right)}
$$

In terms of the variables $(x, y)$, the solution looks as

$$
\Psi_{n}(x, y)=A_{n} \mathrm{e}^{-\alpha\left(x^{2}+y^{2}\right)} \mathrm{e}^{-\lambda(x-i y)}(2 \alpha(x+i y)+\lambda)^{n} .
$$

One must note that $\left[\hat{L}_{z}, \hat{H}\right]=0$, where $\hat{L}_{z}$ is the infinitesimal generator of the element of the group of rotations around z-axis, which is the group of symmetries of our Hamiltonian. Therefore, $\hat{L}_{z} \Psi_{n}$ must be other eigenfunction of the Hamiltonian for the same eigenvalue $E_{n}$. It is not difficult to see that

$$
\hat{L}_{z} \Psi_{n}=\hbar\left(\lambda z^{*}+\frac{2 \alpha n z}{2 \alpha z+\lambda}\right) \Psi_{n},
$$

and that

$$
\hat{H}\left(\hat{L}_{z} \Psi_{n}\right)=E_{n}\left(\hat{L}_{z} \Psi_{n}\right) .
$$

Note that one gets the following expectetion value

$$
\left\langle n\left|r^{2}\right| n\right\rangle=2 A_{n}^{2} \mathrm{e}^{\frac{|\lambda|^{2}}{2 \alpha}}(2 \alpha)^{n-2} \sum_{k=0}^{n}\left(\begin{array}{l}
n \\
k
\end{array}\right) \Gamma\left(n-k+1+\frac{1}{2}\right) \Gamma\left(k+\frac{1}{2}\right)+\frac{|\lambda|^{2}}{4 \alpha^{2}}
$$

which can be used to calculate the area of the surface of a ring of inner radius $r_{1}$ and external radius $r_{2}$ on the plane $\mathrm{x}-\mathrm{y}$, given by $\Delta A=\pi\left(r_{2}^{2}-r_{1}^{2}\right)$ classically, but in our quantum case, one has

$$
\Delta A=\pi\left[\left\langle n\left|r^{2}\right| n\right\rangle-\left\langle n\left|r^{2}\right| n\right\rangle\right]=\frac{\pi}{\alpha} \times\left\{B\left(n_{2}\right)-B\left(n_{1}\right)\right\},
$$

where $B(n)$ has been defined as

$$
B(n)=\frac{\sum_{k=0}^{n}\left(\begin{array}{l}
n \\
k
\end{array}\right) \Gamma\left(n-k+1+\frac{1}{2}\right) \Gamma\left(k+\frac{1}{2}\right)}{\sum_{k=0}^{n}\left(\begin{array}{l}
n \\
k
\end{array}\right) \Gamma\left(n-k+\frac{1}{2}\right) \Gamma\left(k+\frac{1}{2}\right)}
$$


Numerically, one finds that $B(2 n)=(2 n+1) / 2$ and $B(2 n+1)=n+1$ for $n \in \mathcal{Z}$. Therefore, one gets that

$$
B\left(n_{2}\right)-B\left(n_{1}\right)= \begin{cases}n_{2}-n_{1} & \text { even }- \text { even } \\ 2\left(n_{2}-n_{1}\right) & \text { odd }- \text { odd } \\ n_{2}-n_{1}-1 / 2 & \text { eve }- \text { odd } \\ n_{2}-n_{1}+1 / 2 & \text { odd }- \text { even }\end{cases}
$$

Thus, this means that $2 \alpha(\Delta A) / \pi$ is an integer number independently on the integers $n_{1}$ and $n_{2}$, that is (see 12)

$$
\frac{q B(\Delta A)}{2 \pi \hbar c} \in \mathcal{Z},
$$

or

$$
\frac{\Phi}{\Phi_{0}}=j, \quad j \in \mathcal{Z},
$$

where $\Phi$ is the magnetic flux, $\Phi=B(\Delta A)$, and $\Phi_{0}$ is the quantum magnetic flux, $\Phi_{0}=2 \pi \hbar c / q$.

One needs to mention that Laughlin [28] gave a solution to this problem which is equivalent Landau' solution, and this equivalence was demonstrated by Orion [29]. Their solutions are of separable variable type in the polar coordinates $\varphi$ and $\rho$ in the space $x-y(x=\rho \cos \varphi, y=\rho \sin \kappa)$. However, as one can see from (47) or (49), this solution is not of separable variable type in these coordinates, and this is consistent with the fact that the eigenvalue problem (29) written in polar coordinates

$$
-\frac{\hbar^{2}}{2 m}\left\{\frac{1}{\rho} \frac{\partial}{\partial \rho}\left(\rho \frac{\partial \Psi}{\partial \rho}\right)+\frac{1}{\rho^{2}} \frac{\partial^{2} \Psi}{\partial \varphi}\right\}+\frac{q^{2} B^{2}}{8 m c^{2}} \rho^{2} \Psi-i \hbar \frac{q B}{2 m c} \frac{\partial \Psi}{\partial \varphi}=E \Psi,
$$

is not of separable variable type in these coordinates. Therefore, the solution (47) cannot be equivalent to Landau-Laughlin solutions.

\section{Conclusions and Comments}

We have summarized our previous results about the quantization of a charged particle in a flat box and under constants magnetic and electric fields for several electromagnetic static cases using Landau's gauge for the static magnetic field, and using Fourier transformation to solve the linear differential equations resulting from the Shrödinger's equation. We have pointed out again that the full solution obtained is different from Landau's solution for the wave function, but as expected, Landau's levels appear as the solution of the eigenvalues. In all cases, a characteristic phase appears which indicates the non separability on the related variables, which is consistent with the non separability of these variables on the eigenvalue differential equation defined by the Hamiltonian. The quantization of the magnetic flux now appears by considering the number of states between two Landau's levels, and this result is related with the density of states between these levels. Finally, we considered the case for symmetric gauge for the 
static magnetic field on the two dimensional plane, and we have shown that a non separable solution exists which is different to Landau-Laughin solution, and the same Landau's levels are obtained. We keep on considering that the approach given here could be very useful to understand quantum Hall effect and related phenomena mainly, because with our solutions a Hall's voltage appears.

\section{Conflicts of Interest}

The authors declare no conflicts of interest regarding the publication of this paper.

\section{References}

[1] Klitzing, K., Dorda, G. and Pepper, M. (1980) Physical Review Letters, 45, 494-497. https://doi.org/10.1103/PhysRevLett.45.494

[2] Landau, L.D. and Lifshitz, E.M. (2013) Quantum Mechanics: Non-Relativistic Theory, Volume 3. Elsevier, Amsterdam.

[3] Ando, T., Matsumoto, Y. and Uemura, Y. (1975) Journal of the Physical Society of Japan, 39, 279-288. https://doi.org/10.1143/JPSJ.39.279

[4] Laughlin, R.B. (1981) Physical Review B, 23, 5632(R). https://doi.org/10.1103/PhysRevB.23.5632

[5] Halperin, B.I. (1982) Physical Review B, 25, 2185. https://doi.org/10.1103/PhysRevB.25.2185

[6] Laughlin, R.B. (2000) Uspekhi Fizicheskikh Nauk, 170, 292-303. https://doi.org/10.3367/UFNr.0170.200003f.0292

[7] Tsui, D.C., Stormer, H.L. and Gossard, A.C. (1982) Physical Review Letters, 48, 1559-1562. https://doi.org/10.1103/PhysRevLett.48.1559

[8] Laughlin, R.B. (1983) Physical Review Letters, 50, 1395. https://doi.org/10.1103/PhysRevLett.50.1395

[9] Jain, J.K. (1989) Physical Review Letters, 63, 199-202. https://doi.org/10.1103/PhysRevLett.63.199

[10] Konig, M., Wiedmann, S., Brune, C., Roth, A., Buhmann, H., Molenkamp, L.W., Qi, X.-L. and Zhang, S.-C. (2007) Science, 318, 766-770. https://doi.org/10.1126/science.1148047

[11] Zhang, S.-B., Lu, H.-Z. and Shen, S.-Q. (2015) Scientific Reports, 5, 13277, 13279.

[12] MacDonald, A.H. and Streda, P. (1984) Physical Review B, 29, 1616-1619. https://doi.org/10.1103/PhysRevB.29.1616

[13] Yang, Z.H. and Han, J.H. (2011) Physical Review B, 83, Article ID: 045415. https://doi.org/10.1103/PhysRevB.83.045415

[14] MacDonald, A.H., Rice, T.M. and Brinkman, W.F. (1983) Physical Review B, 28, 3648-3650. https://doi.org/10.1103/PhysRevB.28.3648

[15] Fu, L. and Kane, C.L. (2008) Physical Review Letters, 100, Article ID: 096407. https://doi.org/10.1103/PhysRevLett.100.096407

[16] Freedman, M.H., Larsen, M. and Wang, Z.H. (2002) Communications in Mathematical Physics, 227, 605-622. https://doi.org/10.1007/s002200200645

[17] Kitaev, A. (2006) Annals of Physics, 321, 2-111. https://doi.org/10.1016/j.aop.2005.10.005 
[18] Gustavo, J.A.L. and Lopez, V. (2020) Journal of Modern Physics, 11, 1731. https://doi.org/10.4236/jmp.2020.1110106

[19] Prange, R.E. (1981) Physical Review B, 23, 4802-4805. https://doi.org/10.1103/PhysRevB.23.4802

[20] Laughlin, R.B. (1999) Reviews of Modern Physics, 71, 863-874. https://doi.org/10.1103/RevModPhys.71.863

[21] Yoshioka, D. (2013) The Quantum Hall Effect. Volume 133, Springer Science \& Business Media, Berlin.

[22] Datta, S. (1997) Electronic Transport in Mesoscopic Systems. Cambridge University Press, Cambridge.

[23] Messiah, A. (1999) Quantum Mechanics. Dover Publications, Mineola.

[24] Rudin, W. (1974) Functional Analysis. TATA McGraw-Hill Publishing Co., New Delhi.

[25] Deaver, B.S. and Fairbank, W.M. (1961) Physical Review Letters, 7, 43-46. https://doi.org/10.1103/PhysRevLett.7.43

[26] London, F. (1950) Superuids. John Wiley and Sons, New York.

[27] Lebedev, N.N. (1972) Special Function and Their Applications. Dover Publications, Inc., Mineola.

[28] Laughlin, R.B. (1983) Physical Review B, 27, 3383-3389. https://doi.org/10.1103/PhysRevB.27.3383

[29] Ciftja, O. (2020) European Journal of Physics, 41, Article ID: 035404. https://doi.org/10.1088/1361-6404/ab78a7 